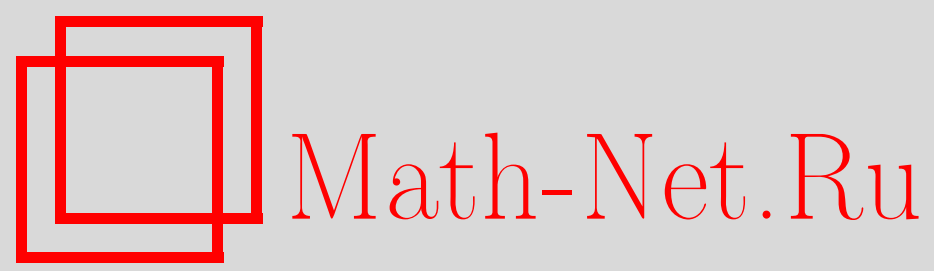

A. E. Lepskiy, Decompositional approach for evaluation of internal conflict in the framework of the evidence theory, Нечеткие системы и мягкие вычисления, 2020, том 15, выпуск 1, 43-63

DOI: https://doi.org/10.26456/fssc63

Использование Общероссийского математического портала Math-Net.Ru подразумевает, что вы прочитали и согласны с пользовательским соглашением

http://www.mathnet.ru/rus/agreement

Параметры загрузки:

IP : 34.229 .45 .116

26 апреля 2023 г., 13:28:07 
UDC 510.676, 519.7

\title{
DECOMPOSITIONAL APPROACH FOR EVALUATION OF INTERNAL CONFLICT IN THE FRAMEWORK OF THE EVIDENCE THEORY ${ }^{1}$
}

Lepskiy A.E.

National Research University Higher School of Economics, Moscow

\begin{abstract}
Received 15.03.2020, revised 15.04.2020.
The concept of conflict is one of the central in the belief functions theory. There are differences between external and internal conflicts. A new method for estimating the internal conflict is proposed and studied in this paper. This method assumes that the original body of evidence was derived from simpler evidence using some combining rule. Therefore, an internal conflict can be considered as an external conflict of decomposition of the original body of evidence. This approach is specified in the article for decomposition by the Dempster rule and decomposition by the disjunctive consensus rule. The possible limits of change of the internal conflict are found in the case of these two combining rules for single-focal (categorical) and two-focal bodies of evidence. The decomposition method is discussed in detail for the case of a universal set with two alternatives.
\end{abstract}

Keywords: conflict measure, belief functions theory, imprecise index, combining rules.

Nechetkie Sistemy $i$ Myagkie Vychisleniya [Fuzzy Systems and Soft Computing], 15:1 (2020), 43-63, https://doi.org/10.26456/fssc63

\section{Introduction}

The concept of conflict is one of the central in the belief functions theory $[7,28]$. The conflict between pieces of evidence characterizes the inconsistency between the sources of information described by these pieces of evidence. There are several approaches to evaluating the conflict between evidence (external conflict). Historically, the first was the measure of conflict associated with the Dempster rule [7]. Currently, measures of conflict are studied in several directions, such as the study of the axiomatics of measures of conflict $[3,8,21]$, the study of ways to specify measures of conflict $[4,12,13,16,20,21,23]$, the study of the geometric interpretation of a conflict measure [5], the application of measures of conflict to various problems of data analysis and decision making. The problem of combining contradictory evidence is considered within framework of conflict management [15,25].

Along with the conflict between a few bodies of evidence, structural conflict in one body of evidence is also considered. Such a conflict is called internal, unlike external conflict between a few bodies of evidence. An example of evidence with an internal

\footnotetext{
${ }^{1}$ The study has been funded by the Russian Academic Excellence Project '5-100'. This work was also partially supported by the grant 18-01-00877 of RFBR (Russian Foundation for Basic Research).
} 
conflict is the following statement: 'shares of the company will be in a week in the interval $[5,15]$ or $[40,50]^{\prime}$. The internal conflict of evidence relates with analysis of the heterogeneity of information source. The heterogeneity of information influences on the decision-making regarding the choice of methods for its processing, reliability, etc. For example, if the evidence has a large internal conflict, then the question arises about belief in such evidence. There are different approaches to evaluation of internal conflict. An overview of these approaches is given in Section 4.

An approach based on the idea of decomposition will be considered and studied in this paper to define an internal conflict. This approach is based on the assumption that evidence with a large internal conflict could have been received by combining information from several sources. If we find a decomposition of the original evidence into simple bodies of evidence, then we will be able to assess the internal conflict as an external conflict of the decomposing set. But we can only estimate the boundaries of the conflict in this way, because decomposition will not be unambiguous in the general case. In the general case the bodies of evidence of a decomposed set should have a simpler structure than the initial evidence. Therefore, these restrictions on the admissible set of decomposing evidence bodies should be taken into account in the formulation of the decomposition problem. The decomposition approach generalizes some other methods for evaluation of internal conflict (for example, [26]). In addition, it is more universal, it allows the use of various restrictions and it has a good interpretation. In this paper we consider only the most general restrictions that are related with the character (optimistic or pessimistic) of combining rules. These restrictions can be given with the help of imprecision indices. Imprecision indices are used for evaluation of changing ignorance after applying combining rules. The necessary information on imprecision indices is given in Section 6 .

In the general case, the evaluation of internal conflict of evidence, is defined as a solution to the optimization problem, and is formulated with some restrictions in Section 5 . The properties of internal conflict estimations by the decomposition method for the Dempster rule and the disjunctive consensus rule are considered in Section 6 and Section 7, respectively. In these sections, only the most general properties with the most general restrictions on the nature of the combining rules are investigated. The choice of combining rules is due to their polar nature. Dempster's rule is optimistic, and the disjunctive consensus rule is pessimistic (see Section 5). The calculation of internal conflict estimates by the decomposition method with stronger restrictions is shown in the examples. The decomposition method is considered in more detail for the case of a set with two alternatives in Section 8. Finally, Section 9 presents some conclusions.

Some results of this paper were announced in $[18,19]$.

\section{Preliminary Concepts of the Belief Functions Theory and External Conflict Measure}

Let $X$ be some set and $\mathcal{X}$ be some finite family of its subsets. In particular, if $X$ be a finite set, then without loss of generality we can assume that $\mathcal{X}=2^{X}$ be the power set of $X$. The so-called mass function is considered on the family $\mathcal{X}$ as a set function $m: \mathcal{X} \rightarrow[0,1]$ such that $\sum_{A \in \mathcal{X}} m(A)=1$ and $m(\emptyset)=0$, if $\emptyset \in \mathcal{X}$. The value $m(A)$ can have different interpretations. In particular, $m(A)$ can characterize the degree of belief that the true alternative from $X$ belongs to the set $A \in \mathcal{X}$.

If $A \in \mathcal{X}$ satisfies the condition $m(A)>0$, then the subset $A$ is called a focal element. Let $\mathcal{A}$ denote the set of all focal elements. Then the pair $F=(\mathcal{A}, m)$ forms 
the so-called body of evidence. Different body structures of evidence are considered in the belief functions theory. The so-called categorical body of evidence (or simply categorical evidence) $F_{A}=(A, 1)$ (i.e. $\mathcal{A}=\{A\}$ and $\left.m(A)=1\right), A \in \mathcal{X}$ is the simplest such structure. In particular, $F_{X}=(X, 1)$ is called vacuous evidence because this evidence is completely non-informative. Through $\mathcal{F}(X)$ we will denote the set of all bodies of evidence on $X$.

It is not difficult to see that the mass functions on the $X$ form a convex set. Therefore, we can consider that the set of bodies of evidence also forms a convex set: $F=(\mathcal{A}, m)=\sum_{j=1}^{n} \alpha_{j} F_{j}$, if $0 \leq \alpha_{j} \leq 1, j=1, \ldots, n, \sum_{j=1}^{n} \alpha_{j}=1$, where $m(A)=\sum_{j=1}^{n} \alpha_{j} m_{j}(A), \mathcal{A}=\bigcup_{j=1}^{n} \mathcal{A}_{j}$ for $F_{j}=\left(\mathcal{A}_{j}, m_{j}\right) \in \mathcal{F}(X), j=1, \ldots, n$. In this case any evidence can be presented as a convex linear combination of categorical bodies of evidence: $F=(\mathcal{A}, m)=\sum_{A \in \mathcal{A}} m(A) F_{A}$.

The body of evidence of the form $F_{A}^{\alpha}=(1-\alpha) F_{A}+\alpha F_{X}, \alpha \in[0,1]$ is called a simple evidence. If the body of evidence $F=(\mathcal{A}, m)$ satisfies the condition $A \subseteq B$ or $B \subseteq A$ for all $A, B \in \mathcal{A}$, then this evidence is called consonant.

Two main functions are related to the mass function of the body of evidence $F=(\mathcal{A}, m)$. There are the belief function [28] $\operatorname{Bel}(B)=\sum_{A \subseteq B} m(A)$ and the plausibility function $\operatorname{Pl}(B)=1-\operatorname{Bel}(\neg B)=\sum_{A \cap B \neq \emptyset} m(A)$. These functions define the lower and upper probability estimates of an $B$ event, respectively.

If $\mathcal{B}(X)$ be a set of all belief functions on $X$, then there is a one-to-one correspondence between the sets $\mathcal{F}(X)$ and $\mathcal{B}(X)$, which will be denoted as follows: $\mathcal{F}(X) \ni F=(\mathcal{A}, m) \leftrightarrow B e l \in \mathcal{B}(X)$, where $\operatorname{Bel}(B)=\sum_{A \subseteq B, A \in \mathcal{A}} m(A)$.

Suppose we have information from two sources in the form of two bodies of evidence $F_{1}=\left(\mathcal{A}_{1}, m_{1}\right)$ and $F_{2}=\left(\mathcal{A}_{2}, m_{2}\right)$. Then for solving some problems we need to measure the conflict (contradiction) between these pieces of evidence. There are many different approaches to assessing external conflict between two sources of information $[12,13,16,20,21]$. In this article we will use the most popular measure of external conflict

$$
K_{0}=K_{0}\left(F_{1}, F_{2}\right)=\sum_{\substack{B \cap C=\emptyset \\ B \in \mathcal{A}_{1}, C \in \mathcal{A}_{2}}} m_{1}(B) m_{2}(C) .
$$

It is not difficult to see that $K_{0} \in[0,1]$ and $K_{0}=0$ in the case $B \cap C \neq \emptyset$ for all $B \in \mathcal{A}_{1}, C \in \mathcal{A}_{2}$ (absolutely non-conflict situation); $K_{0}=1$ in the case $A \cap B=\emptyset$ for all $A \in \mathcal{A}_{1}, B \in \mathcal{A}_{2}$ (absolutely conflict situation).

This measure of external conflict is related to Dempster's rule, which is defined by $m_{D}(A)=\frac{1}{1-K_{0}} \sum_{B \cap C=A} m_{1}(B) m_{2}(C), \forall A \neq \emptyset, m_{D}(\emptyset)=0$, if $K_{0} \neq 1$. Dempster's rule cannot be applied in the case $K_{0}=1$.

The choice of this measure to evaluate of external conflict is due to its popularity, simplicity and good interpretability. This measure is generalized for an arbitrary set of bodies of evidence

$$
K_{0}\left(F_{1}, \ldots, F_{k}\right)=\sum_{\substack{A_{1} \cap \ldots \cap \cap A_{k}=\emptyset, A_{s} \in \mathcal{A}_{s}, s=1, \ldots, k}} m_{1}\left(A_{1}\right) \ldots m_{k}\left(A_{k}\right),
$$

where $F_{s}=\left(\mathcal{A}_{s}, m_{s}\right), \quad s=1, \ldots, k$. Dempster's rule is an optimistic rule in the following sense. If one source indicates $x \in A$ and another source indicates $x \in B$, and $A \cap B \neq \emptyset$ (in other words, we have two categorical evidence $F_{A}$ and $F_{B}$ ), then we will receive a new categorical evidence $F_{A \cap B}$ after combining according to Dempster's rule that means $x \in A \cap B$. 
The following disjunctive consensus rule [10] is the "opposite"of Dempster's rule:

$$
m_{D P}(A)=\sum_{B \cup C=A} m_{1}(B) m_{2}(C), \quad A \in 2^{X} .
$$

The disjunctive consensus rule is a pessimistic rule. If one source indicates $x \in A$ and another source indicates $x \in B$, then we will receive a new categorical evidence $F_{A \cup B}$ after combining according to the disjunctive consensus rule that means $x \in A \cup B$.

A description of the other rules for combining bodies of evidence can be found in [27]. Some general combination schemes are discussed in [17].

\section{The Main Approaches to Evaluation of Internal Conflict}

The following approaches can be distinguished to evaluate of internal conflict.

1. Entropy approach. This is the first approach to the study of internal conflict, which was considered at the turn of the $80 \mathrm{~s}$ of the last century. In this case, the measure of internal conflict should reflect the distribution of the mass function of the evidence on the 'conflicting' focal elements. Various measures were proposed to model this distribution: measure of dissonance, measure of confusion, measure of discord, measure of strife etc. [14]. For example, measure of dissonance was defined as [30]

$$
E(m)=-\sum_{A \in \mathcal{A}} m(A) \log _{2}(1-K(A))
$$

where $K(A)=\sum_{A \cap B=\emptyset} m(B)$ is the total value of the masses of focal elements conflicting with the $A$ with respect to the ratio of 'non-intersection'. Then $E(m)$ is the average value of conflicting focal elements with respect to the ratio of 'nonintersection'.

2. Auto-conflict approach. If we have $K_{0}(F, F)=0$, then the body of evidence $F=(\mathcal{A}, m)$ can be considered without internal conflict. Therefore, the value $K_{\text {aut }}(F)=K_{0}(F, F)$ can be considered as a measure of internal conflict. This approach was considered in [22]. If $K_{0}(F, F)=0$ then $\Omega_{\mathcal{A}}=\bigcap_{A \in \mathcal{A}} A \neq \emptyset$ and $P l(x)=$ $\sum_{A \in \mathcal{A}: x \in A} m(A)=1 \forall x \in \Omega_{\mathcal{A}}$. Therefore, the following measure of internal conflict was introduced in [6]: $K_{p l}(F)=1-\max \{P l(x): x \in X\}$.

3. Decompositional approach. The decomposition approach is based on the assumption that a large internal conflict may be caused in some cases by the heterogeneity of information sources. In other words, the analyzed evidence could be obtained by the aggregation of other more simple bodies of evidence. For example, in [26] internal conflict was defined as a conflict among the so-called generalized simple bodies of evidence (that is, the body of evidence of the form $F_{A}^{\alpha}=(1-\alpha) F_{A}+\alpha F_{X}$ with $\left.\alpha \in(0, \infty)\right)$ on which the original evidence decomposes uniquely. A decompositional approach to assessing the internal conflict of evidence close to [26] was also considered in [24]. But such expansions do not always have a clear interpretation. In addition, the analyzed body of evidence could be obtained by combining not simple bodies of evidence. For example, these may be consonant (but not simple) bodies of evidence.

Various restrictions can be considered in the decompositional approach. For example, the structural constraint, restrictions on the conflict of decomposable evidence, restrictions on the proximity to the precedents etc. 
Below we consider the decomposition approach, in which the restrictions on the decomposable set are related to the nature of the combining rules.

\section{Decomposition of the Body of Evidence}

Suppose that the body of evidence $F=(\mathcal{A}, m)$ was obtained by aggregating several other simpler bodies of evidence. For example, weather forecasts are based on aggregating several other forecasts. In other words, the analyzed body of evidence $F=(\mathcal{A}, m)$ was obtained as a result of applying of some combining rule $R$ to more simple bodies of evidence $F_{i}=\left(\mathcal{A}_{i}, m_{i}\right) \in \mathcal{F}(X), i=1, \ldots, l: F=R\left(F_{1}, \ldots, F_{l}\right)$. If we find these bodies of evidence, then we can evaluate the conflict between them with the help of some measure of external conflict $K$. Then this value can be considered as an internal conflict of the original evidence: $K_{i n}(F)=K\left(F_{1}, \ldots, F_{l}\right)$ with constraint $F=R\left(F_{1}, \ldots, F_{l}\right)$. In the general case, this problem has many solutions. Therefore, it makes sense to formulate optimization problems of finding the smallest $\underline{K}_{i n}^{R}(F)$ and largest $\bar{K}_{i n}^{R}(F)$ conflict values:

$$
\underline{K}_{i n}^{R}(F)=\inf _{F=R\left(F_{1}, \ldots, F_{l}\right)} K\left(F_{1}, \ldots, F_{l}\right), \quad \bar{K}_{i n}^{R}(F)=\sup _{F=R\left(F_{1}, \ldots, F_{l}\right)} K\left(F_{1}, \ldots, F_{l}\right) .
$$

We denote by $S_{n}=\left\{\mathbf{s}=\left(s_{i}\right)_{i=1}^{n}: s_{i} \geq 0 \forall i, \sum_{i=1}^{n} s_{i}=1\right\}$ a $n$-dimensional simplex.

Below we use Dempster's and the disjunctive consensus rules for decomposition and we use only the measure from Dempster's rule to evaluate of external conflict after the decomposition. The choice of these rules is due to the fact that Dempster's and the disjunctive consensus rules are extreme cases of combining from the point of view of belief in the reliability of information sources. The choice measure to evaluate of external conflict is due to its popularity, simplicity and good interpretability. In addition, we consider below the case of decomposition into two bodies of evidence. In general, the choice of the number of bodies for decomposition is carried out by the method of enumeration.

\subsection{Decomposition of the body of evidence according to Dempster's rule}

If we use Dempster's rule $R=D$ for combining, then problems (3) for $l=2$ take the form. We need to find

$$
K_{0}\left(F_{1}, F_{2}\right)=\sum_{\substack{B \cap C=\emptyset, B \in \mathcal{A}_{1}, C \in \mathcal{A}_{2}}} m_{1}(B) m_{2}(C) \rightarrow \sup \quad \text { (inf) }
$$

with constraints

$$
\begin{gathered}
\mathbf{m}_{1}=\left(m_{1}(B)\right)_{B \in \mathcal{A}_{1} \in S_{\left|\mathcal{A}_{1}\right|}, \mathbf{m}_{2}=\left(m_{2}(C)\right)_{C \in \mathcal{A}_{2} \in S_{\left|\mathcal{A}_{2}\right|},}}\left(1-K_{0}\left(F_{1}, F_{2}\right)\right) m(A)=\sum_{\substack{B \cap C=A, B \in \mathcal{A}_{1}, C \in \mathcal{A}_{2}}} m_{1}(B) m_{2}(C), A \in \mathcal{A}, \\
K_{0}\left(F_{1}, F_{2}\right)<1 .
\end{gathered}
$$


The conditions (6)-(7) are quadratic, and the condition (5) is linear. Therefore, in general, this is a quadratic optimization problem. But in the general case, the problem (4)-(7) has only a trivial solution. Indeed, we have $\underline{K}_{i n}^{D}(F)=K_{0}\left(F, F_{X}\right)=0$. In addition, we have $K_{0}\left(F_{1}, F_{2}\right)=1$, if bodies of evidence $F_{i}=\left(\mathcal{A}_{i}, m_{i}\right) \in \mathcal{F}(X)$, $i=1,2$ satisfy the condition $B \cap C=\emptyset \quad \forall B \in \mathcal{A}_{1}, \forall C \in \mathcal{A}_{2}$. These pieces of evidence satisfy the conditions (5)-(6) (without (7)), but they are not related to the original evidence $F$.

\subsection{Decomposition of the body of evidence according to the disjunctive consensus rule}

If we use the disjunctive consensus rule $R=D P$ for combining, then the problem of finding an internal conflict by decomposition method is reduced to solving an optimization problem (4) with constraints (2), (5).

Sometimes when using disjunctive consensus rule, it is convenient to assume that the empty set can be a focal element, as it is assumed in the transferable belief model (TBM) [29]. The value $m(\emptyset)$ is the degree of confidence that $x \notin X$. In this case we will denote by $\underline{\mathcal{K}}_{i n}^{D P}(F)$ and $\overline{\mathcal{K}}_{i n}^{D P}(F)$ the corresponding solutions of the optimization problems $(4),(2),(5)$. We will call this solution the generalized solution of the decomposition problem. But then we have $\overline{\mathcal{K}}_{i n}^{D P}(F)=K_{0}\left(F, F_{\emptyset}\right)=1$. Thus, the problem of finding $\bar{K}_{i n}^{D}(F)$ and $\underline{K}_{i n}^{D}(F)\left(\right.$ or $\underline{\mathcal{K}}_{i n}^{D P}(F)$ and $\left.\overline{\mathcal{K}}_{i n}^{D P}(F)\right)$ in such a general formulation has only trivial solutions.

However, decomposition cannot be arbitrary in the task of evaluating an internal conflict. The body of evidence into which the original body of evidence is decomposed should be simple in some sense. In this article we will consider only the limitations associated with the nature of the combining rules. For example, it is known that Dempster's rule and the disjunctive consensus rule are optimistic and pessimistic, respectively. The restrictions on the set of admissible bodies of evidence agreeing with the character of combining rules can be set using imprecision indices considered in the next section.

\section{Imprecision Indices and Change of Ignorance after Using of Combining Rule}

\subsection{Imprecision Indices}

Assume that a true alternative belongs to the non empty set $A \subseteq X$. This event is described by the body of evidence $F_{A}=(A, 1)$. It is known that the degree of uncertainty of this event can be estimated using Hartley's measure $H\left(F_{A}\right)=\log _{2}|A|$. For belief structure $F=(\mathcal{A}, m) \in \mathcal{F}(X)$ Hartley's measure can be generalized as follows (so called the generalized Hartley's measure $[9,11]$ ):

$$
f(F)=\sum_{A \in \mathcal{A}} m(A) \log _{2}|A|
$$

We will be consider the following generalization of this construction [2].

Definition 1. The functional $f: \mathcal{F}(X) \rightarrow[0,1]$ will be called the imprecision index if it satisfies the conditions: 
1. $f(F)=0 \forall F=(\mathcal{A}, m) \in \mathcal{F}(X):|A|=1 \forall A \in \mathcal{A}$; in other words $f(F)=0$, if $F \leftrightarrow p$, where $p$ is a probability measure;

2. $f\left(F_{1}\right) \geq f\left(F_{2}\right) \forall F_{1}, F_{2} \in \mathcal{F}(X)$, where $F_{i} \leftrightarrow g_{i}, i=1,2$ and $g_{1} \leq g_{2}$ (i.e. $\left.g_{1}(A) \leq g_{2}(A) \forall A \subseteq X\right)$;

3. $f\left(F_{X}\right)=1$.

Sometimes it is important that conditions (1), (3) of the Definition 1 are strictly fulfilled (let us call such imprecision indices strict), i.e. the following conditions should be met instead of 1) and 3): (1') $f(F)=0 \Leftrightarrow F \leftrightarrow p$, where $p$ is a probability measure; (3') $f(F)=1 \Leftrightarrow F=F_{X}$.

The class of linear indices is the simplest and most important family of imprecision indices. An linear imprecision index must satisfy the condition: $f\left(\sum_{j=1}^{n} \alpha_{j} F_{j}\right)=$ $\sum_{j=1}^{n} \alpha_{j} f\left(F_{j}\right)$ for any $F_{j} \in \mathcal{F}(X), j=1, \ldots, n$ and $\left(\alpha_{j}\right) \in S_{n}$.

The linear functional $f$ on $\mathcal{F}(X)$ is uniquely determined by its values on categorical evidence $\left\{F_{B}\right\}_{B \neq \emptyset}$. The values of the functional $f$ on $\left\{F_{B}\right\}_{B \neq \emptyset}$ denoted by $\mu_{f}(B)$, i.e. $\mu_{f}(B)=f\left(F_{B}\right), B \neq \emptyset$. In addition, it is convenient to assume that $\mu_{f}(\emptyset)=0$ (in the framework of the TBM approach, this means that $f\left(F_{\emptyset}\right)=0$ ). Then it is not difficult to see that the linear imprecision index $f$ is a convex combination of values of set function $\mu_{f}$. More precisely the following proposition is true [2].

Proposition 1. Formula

$$
f(F)=\sum_{B \in \mathcal{A}} m(B) \mu_{f}(B), \quad F=(\mathcal{A}, m),
$$

is a general representation of a linear imprecision index $f$ on $\mathcal{F}(X)$ iff the following conditions are true: 1) $\mu_{f}(\{x\})=0 \forall x \in X$; 2) $\mu_{f}(X)=f\left(F_{X}\right)=1$; 3) $\sum_{B: A \subseteq B}(-1)^{|B \backslash A|} \mu_{f}(B) \leq 0 \forall A \neq \emptyset, X$.

Note that conditions 1) and 2) in the Proposition 1 should be replaced by conditions 1') $\mu_{f}(B)=0 \Leftrightarrow|B|=1$ and 2') $\mu_{f}(B)=1 \Leftrightarrow B=X$ in the case of a strict linear imprecision index.

Example 1. Let $\mu_{f}(B)=\psi(|B|)$. Then functions $\psi(t)=\ln t / \ln |X|$, $\psi(t)=(t-1)^{\alpha} /(|X|-1)^{\alpha}, \alpha \in(0,1]$ satisfy the conditions 1$\left.)-3\right)$ of Proposition 1 (and $\left.\left.1^{\prime}\right), 2^{\prime}\right)$ ) and corresponding functional (8) will be a strict linear imprecision index. In particular the normalized generalized Hartley's measure is a strict linear imprecision index on $\mathcal{F}(X)$.

The value $f(F)$ characterizes the amount of ignorance in the information contained in evidence $F$.

\subsection{Change of Ignorance after Using the Combining Rule}

Let two sources of information be described by two categorical bodies of evidence $F_{A}$ and $F_{B}$ respectively, where $A, B \in 2^{X} \backslash\{\emptyset\}$. The first source claims that the true alternative is contained in the set $A$, and the second source claims that the true alternative is contained in the set $B$. If we apply the disjunctive consensus rule for these categorical bodies of evidence then we get $D P\left(F_{A}, F_{B}\right)=F_{A \cup B}$. In other words, we get the evidence that a true alternative is contained in the set $A \cup B$. 
This evidence is pessimistic, since the uncertainty after the combination has not diminished. Indeed, for a linear imprecision index, the following inequality is true $f\left(F_{A \cup B}\right)=\mu_{f}(A \cup B) \geq \mu_{f}(A)=f\left(F_{A}\right)$ (and similarly for $f\left(F_{B}\right)$ ).

The opposite result is observed if we apply Dempster's rule to the same evidence bodies: $D\left(F_{A}, F_{B}\right)=F_{A \cap B}$ for $A \cap B \neq \emptyset$ (if $A \cap B=\emptyset$, then $K_{0}=1$ and Dempster's rule cannot be applied). This evidence is optimistic, since the uncertainty after the combination has not increased: $f\left(F_{A \cap B}\right)=\mu_{f}(A \cap B) \leq f\left(F_{A}\right)$.

The following propositions summarize the above arguments (see for details [17]).

Proposition 2. If $F=D P\left(F_{1}, F_{2}\right), F_{1}, F_{2} \in \mathcal{F}(X)$, then we have $f(F) \geq f\left(F_{i}\right)$, $i=1,2$ for any linear imprecision index $f$.

In other words, aggregation using the disjunctive consensus rule does not reduce the uncertainty of the evidence.

Proposition 3. If we have $K_{0}\left(F_{1}, F_{2}\right)=0$ for $F_{1}, F_{2} \in \mathcal{F}(X)$ and $F=D\left(F_{1}, F_{2}\right)$, then the following estimates hold $f(F) \leq f\left(F_{i}\right), i=1,2$ for any linear imprecision index $f$.

In other words, aggregation of non-conflicting bodies of evidence using Dempster's rule does not increase the uncertainty of the evidence.

Below in this paper we will consider only Dempster's and the disjunctive consensus rules for decomposition. The choice of these rules is due to the fact that Dempster's and the disjunctive consensus rules are extreme cases of combining from the point of view of belief in the reliability of information sources.

\section{Decomposition Approach with Dempster's Rule}

\subsection{The General Formulation of the Problem of Evaluation of the Internal Conflict by Decomposition with Dempster's Rule}

Now, the problem of estimating the internal conflict of evidence $F$ in the case of its decomposition into two bodies of evidence $F_{i}=\left(\mathcal{A}_{i}, m_{i}\right) \in \mathcal{F}(X), i=1,2$, can be formulated as follows with taking into account the optimism of Dempster's rule (Proposition 3). We have to find the largest and smallest values of conflict measure $K_{0}\left(F_{1}, F_{2}\right)$ on restrictions $(5)-(7)$ and conditions:

$$
f(F) \leq f\left(F_{i}\right), \quad i=1,2,
$$

where $f: \mathcal{F}(X) \rightarrow[0,1]$ is an imprecision index. Note that $\underline{K}_{i n}^{D}(F)=0$ is always true, since this value is achieved when decomposing into bodies of evidence $F_{1}=F$ and $F_{2}=F_{X}$, and (9) is true. Therefore, we will be interested only in the problem of finding the greatest conflict (4) with constraints (5)-(7), (9).

The upper limitations on the amount of ignorance in the information contained in the decomposable bodies of evidence can be seen in this way: $f\left(F_{i}\right) \leq f_{\max }, i=1,2$, where $f_{\max }$ is a maximum allowable value of ignorance. But we will not consider such restrictions in this paper.

Sometimes the type of initial body of evidence determines the type of body of evidence into which it should be decomposed. 
Example 2. Suppose we have the following body of evidence

$$
F=m_{0} F_{X}+\sum_{i=1}^{n} m_{i} F_{\left\{x_{i}\right\}}, \mathbf{m}=\left(m_{i}\right)_{i=0}^{n} \in S_{n+1}, m_{0} \neq 0 .
$$

We want to evaluate the largest internal conflict of this evidence by the decomposition method on the assumption that Dempster's rule is used for the decomposition. Then it is not difficult to see that the bodies of evidence of decomposition must have the following form: $F_{1}=\alpha_{0} F_{X}+\sum_{i=1}^{n} \alpha_{i} F_{\left\{x_{i}\right\}}, F_{2}=\beta_{0} F_{X}+\sum_{i=1}^{n} \beta_{i} F_{\left\{x_{i}\right\}}$. Then

$$
\begin{array}{r}
K_{0}\left(F_{1}, F_{2}\right)=\sum_{\substack{B \cap C=\emptyset \\
B \in \mathcal{A}_{1}, C \in \mathcal{A}_{2}}} m_{1}(B) m_{2}(C)= \\
=\sum_{i=1}^{n} \sum_{\substack{j=1, i \neq j}}^{n} \alpha_{i} \beta_{j}=\left(1-\alpha_{0}\right)\left(1-\beta_{0}\right)-\sum_{i=1}^{n} \alpha_{i} \beta_{i} \longrightarrow \text { sup }
\end{array}
$$

with constraints

$$
\alpha=\left(\alpha_{i}\right)_{i=0}^{n} \in S_{n+1}, \quad \beta=\left(\beta_{i}\right)_{i=0}^{n} \in S_{n+1},
$$

$$
\begin{gathered}
\left(1-\left(1-\alpha_{0}\right)\left(1-\beta_{0}\right)+\sum_{i=1}^{n} \alpha_{i} \beta_{i}\right) m_{i}=\alpha_{i} \beta_{i}+\alpha_{i} \beta_{0}+\alpha_{0} \beta_{i}, \quad i=1, \ldots, n, \\
\left(1-\left(1-\alpha_{0}\right)\left(1-\beta_{0}\right)+\sum_{i=1}^{n} \alpha_{i} \beta_{i}\right) m_{0}=\alpha_{0} \beta_{0} \\
\alpha_{0} \beta_{0}<\alpha_{0}+\beta_{0}+\sum_{i=1}^{n} \alpha_{i} \beta_{i} .
\end{gathered}
$$

Conditions (11) and (12) imply the equation (13). Inequalities (9) have the form

$$
m_{0} \leq \alpha_{0}, \quad m_{0} \leq \beta_{0} .
$$

We have an optimization problem of finding the largest value of the function (10) with constraints (11), (12), (14), (15).

Other restrictions on the form of decomposed bodies of evidence can also be used in the problem of evaluating an internal conflict. These restrictions may be related to uncertainty of the bodies of evidence (see above), form of evidence (for example, all bodies of evidence in decomposition should be simple or consonant), number of focal elements in evidence bodies, etc. Consider the following example.

Example 3. Suppose we have the following body of evidence $F=m_{1} F_{\left\{x_{1}, x_{2}\right\}}+m_{2} F_{\left\{x_{3}, x_{4}\right\}}+\left(1-m_{1}-m_{2}\right) F_{\left\{x_{2}, x_{3}, x_{4}\right\}}, m_{i} \in(0,1), i=1,2$, $m_{1}+m_{2} \leq 1$ defined on the set $X=\left\{x_{1}, x_{2}, x_{3}, x_{4}\right\}$. Let us estimate the internal conflict of this evidence by decomposition with Dempster's rule, provided that there should be two decomposing bodies of evidence with two focal elements. It is easy to see that this problem may only have a solution of the form $F=D\left(F_{1}, F_{2}\right)$, where

$$
F_{1}=\alpha F_{\left\{x_{1}, x_{2}\right\}}+(1-\alpha) F_{\left\{x_{2}, x_{3}, x_{4}\right\}}, \quad F_{2}=\beta F_{\left\{x_{3}, x_{4}\right\}}+(1-\beta) F_{X}, \quad \alpha, \beta \in(0,1) .
$$


Then the conditions (5)-(7) are equivalent to the system

$$
\left\{\begin{array}{l}
\alpha(1-\beta)=m_{1}(1-\alpha \beta), \\
\beta(1-\alpha)=m_{2}(1-\alpha \beta), \\
\alpha, \beta \in(0,1)
\end{array}\right.
$$

This system has a solution $\alpha=\frac{m_{1}}{1-m_{2}}$ and $\beta=\frac{m_{2}}{1-m_{1}}$. If we use the normalized generalized Hartley's measure (see Subsection ) as an imprecision index, then we get $f(F)=\frac{1}{2}\left(m_{1}+m_{2}\right)+\frac{1}{2}\left(1-m_{1}-m_{2}\right) \log _{2} 3, f\left(F_{1}\right)=\frac{1}{2} \alpha+\frac{1}{2}(1-\alpha) \log _{2} 3$, $f\left(F_{2}\right)=1-\frac{1}{2} \beta$. Then inequalities (9) are satisfied iff $m_{1}+m_{2} \leq 1$, i.e. they will always be fulfilled. In this case, the decomposition measure of the internal conflict will be equal to (compare with the result of Proposition 12 below)

$$
K_{i n}^{D}(F)=\frac{m_{1} m_{2}}{\left(1-m_{1}\right)\left(1-m_{2}\right)},
$$

where $m_{i} \in(0,1), i=1,2, m_{1}+m_{2}<1$. It is interesting to compare this result with other ways of calculating the internal conflict (see Section ). For example, the measure of auto-conflict will be equal to $K_{a u t}(F)=K_{0}(F, F)=m_{1} m_{2}$, and the measure based on the plausibility function will be equal to $K_{p l}(F)=1-\max _{1 \leq i \leq 4} P l\left(x_{i}\right)=1-\max \left\{m_{1}, 1-m_{1}, 1-m_{2}\right\}=\min \left\{m_{1}, m_{2}\right\}$. Thus, we have $K_{\text {in }}^{D}(\bar{F})>K_{a u t}(F), K_{p l}(F)>K_{a u t}(F)$. Besides, we have $K_{\text {in }}^{D}(F)=K_{p l}(F) \Leftrightarrow \max \left\{m_{1}, m_{2}\right\}=\left(1-m_{1}\right)\left(1-m_{2}\right)$.

Let us establish some general properties of the internal conflict found by the decomposition method using Dempster's rule.

\subsection{The Internal Conflict of Categorical Evidence}

Recall that $\underline{K}_{i n}^{D}(F)=0$ for every $F=(\mathcal{A}, m) \in \mathcal{F}(X)$. Consider first the vacuous evidence $F_{X}=(X, 1)$.

Proposition 4. We have $\underline{K}_{i n}^{D}\left(F_{X}\right)=\bar{K}_{i n}^{D}\left(F_{X}\right)=0$ for any strict imprecise index $f$.

Proof. We have $f\left(F_{i}\right)=1, i=1,2$, for body of evidence $F_{X}=(X, 1)$ from condition (9). Further we have $F_{1}=F_{2}=F_{X}$ since an imprecise index $f$ is a strict imprecise index. Then all the conditions of the optimization problem (5)-(7) are fulfilled for pair $\left(F_{X}, F_{X}\right)$ and $K_{0}\left(F_{X}, F_{X}\right)=0$.

For arbitrary categorical body of evidence $F_{A}=(A, 1), A \in 2^{X} \backslash\{\emptyset, X\}$, the upper bound is not always zero, as the following proposition shows.

Proposition 5. If $A \in 2^{X} \backslash\{\emptyset, X\}$, then we have the following estimation for linear imprecision index $f$ :

$$
\bar{K}_{i n}^{D}\left(F_{A}\right) \geq \sup \left\{\sum_{j=1}^{m} \beta_{j}\right\},
$$

where supremum is considered for all $\left(\beta_{j}\right)_{1}^{m}: 0 \leq \beta_{j} \leq 1, j=1, \ldots, m, \sum_{j=1}^{m} \beta_{j}<1$, satisfying condition $\sum_{j=1}^{m} \beta_{j}\left(1-\mu_{f}\left(B_{j}\right)\right) \leq 1-\mu_{f}(A)$ for such a system of subsets $\left\{B_{j}\right\}_{j=1}^{m}$, for which $A \cap B_{j}=\emptyset \forall j=1, \ldots, m$. 
Proof. We will seek the decomposition of evidence $F_{A}=(A, 1)$ in the form $F_{A}=D\left(F_{1}, F_{2}\right)$, where $F_{1}=F_{A}, F_{2}=\sum_{j=1}^{m} \beta_{j} F_{B_{j}}+\beta_{0} F_{X}, A \cap B_{j}=\emptyset$ $\forall j=1, \ldots, m,\left(\beta_{j}\right)_{0}^{m} \in S_{m+1}$. Then $K_{0}=K_{0}\left(F_{1}, F_{2}\right)=\sum_{j=1}^{m} \beta_{j}=1-\beta_{0}$ and conditions (6), (7) are fulfilled, if $\beta_{0}>0$. Condition (9) is equivalent to inequality $\mu_{f}(A) \leq \sum_{j=1}^{m} \beta_{j} \mu_{f}\left(B_{j}\right)+\beta_{0}$. The last inequality, taking into account that $\sum_{j=1}^{m} \beta_{j}=1-\beta_{0}$, implies $\sum_{j=1}^{m} \beta_{j}\left(1-\mu_{f}\left(B_{j}\right)\right) \leq 1-\mu_{f}(A)$. Then $\bar{K}_{\text {in }}^{D}\left(F_{A}\right) \geq \sup \left\{\sum_{j=1}^{m} \beta_{j}\right\}$, where the supremum is taken over all $\left(\beta_{j}\right)_{1}^{m}$, satisfying all conditions of the proposition.

Corollary 1. If $A \in 2^{X} \backslash\{\emptyset, X\}$, then we have the following estimation for linear imprecision index $f$

$$
\bar{K}_{\text {in }}^{D}\left(F_{A}\right) \geq \sup \left\{\beta \in[0,1): \beta\left(1-\mu_{f}(X \backslash A)\right) \leq 1-\mu_{f}(A)\right\} .
$$

Proof. It is enough to put $m=1$ and $B_{1}=X \backslash A$ in the Proposition 5.

Remark 1 . The last inequality can be written as

$$
\bar{K}_{i n}^{D}\left(F_{A}\right) \geq \min \left\{1, \frac{1-\mu_{f}(A)}{1-\mu_{f}(X \backslash A)}\right\},
$$

under the assumption that $\frac{0}{0}=1$.

Corollary 2. We have $\bar{K}_{\text {in }}^{D}\left(F_{A}\right)=1$, if $A \in 2^{X} \backslash\{\emptyset, X\}$ satisfies the condition $\left.\mu_{f}(A) \leq \mu_{f}(X \backslash A)\right)$ for linear imprecision index $f$.

Dirac's measure is a limiting case of categorical evidence satisfying the Corollary 2. Therefore the following statement is true.

Corollary 3. We have $\bar{K}_{i n}^{D}\left(F_{\{x\}}\right)=1$ for every $x \in X$.

Corollary 2 describes the basic relationship between the internal conflict and the informative uncertainty of the evidence under the assumption that the internal conflict is estimated by the decomposition method using Dempster's rule. The functional $\mu_{f}(A)=f\left(F_{A}\right)$ characterizes the information uncertainty of categorical evidence of $F_{A}, A \neq \emptyset$. For example, the normalized Hartley measure $\mu_{f}(A)=\ln |A| / \ln |X|$ may be such a functional. Then Corollary 2 states that the smaller the uncertainty of the information, the greater the uncertainty of the internal conflict of evidence. This is the principle of uncertainty for conflict and information uncertainty. Consider the following example.

Example 4. Let an expert predict the value of shares of a certain company in the segment $A=[50,55]$, while $X=[0,100]$ is a segment of possible changes in the value of shares. Then we have categorical evidence $F_{A}$. The information uncertainty of this evidence is small compared to the information uncertainty of the evidence $F_{X \backslash A}$ regarding (for example) the normalized Hartley measure $\mu_{f}(A)=\ln |A| / \ln |X|$, where $|[a, b]|=b-a+1$. Categorical evidence $F_{A}$ can be obtained by combining, according to Dempster's rule, two bodies of evidence $F_{1}=(1-\varepsilon) F_{[0,50)}+\varepsilon F_{[50,55]}$ and $F_{2}=\varepsilon F_{[50,55]}+(1-\varepsilon) F_{(55,100]}: F_{A}=D\left(F_{1}, F_{2}\right), \varepsilon \in(0,1)$. In this case, the condition (9) is satisfied, and we have $K_{0}\left(F_{1}, F_{2}\right)=(1-\varepsilon)^{2}$. Therefore $\bar{K}_{i n}^{D}\left(F_{A}\right)=1$. If the information uncertainty of the evidence $F_{A}$ would be large, it could not be obtained by a similar combination with the preservation of the condition (9). 


\subsection{The Internal Conflict of Bifocal Evidence}

Next, we consider the internal conflict found by the decomposition method with Dempster's rule for the following body of evidence

$$
F=m F_{A}+(1-m) F_{B}, \quad A, B \neq \emptyset, \quad m \in(0,1) .
$$

We will call such evidence a bifocal evidence. It is clear that the internal conflict of such evidence will depend on the relative position of the focal elements $A$ and $B$. First we consider the case of disjoint focal elements.

Proposition 6. We have $\bar{K}_{i n}^{D}\left(m F_{A}+(1-m) F_{B}\right)=1$ for linear imprecision index $f$, if $A \cap B=\emptyset$.

Proof. Let $F=m F_{A}+(1-m) F_{B}$, where $A \cap B=\emptyset$ and $m \in(0,1)$. We will seek the decomposition of evidence $F$ in the form $F=D\left(F_{1}, F_{2}\right)$, where $F_{1}=\alpha F_{A}+(1-\alpha) F_{B}$, $F_{2}=\beta_{1} F_{A}+\beta_{2} F_{B}+\left(1-\beta_{1}-\beta_{2}\right) F_{A \cup B}, \beta_{1}, \beta_{2}, \alpha \in[0,1], \beta_{1}+\beta_{2} \leq 1$. Then

$$
K_{0}=K_{0}\left(F_{1}, F_{2}\right)=\alpha \beta_{2}+(1-\alpha) \beta_{1}
$$

and the conditions (5)- (7) are equivalent to

$$
\left\{\begin{array}{l}
\left(1-K_{0}\right) m=\alpha\left(1-\beta_{2}\right) \\
\left(1-K_{0}\right)(1-m)=(1-\alpha)\left(1-\beta_{1}\right) \\
\alpha, \beta_{1}, \beta_{2} \in[0,1], \beta_{1}+\beta_{2} \leq 1, K_{0}<1 .
\end{array}\right.
$$

Clearly, (16) implies that $\alpha \in(0,1)$ and $\beta_{1}, \beta_{2} \neq 1$ (otherwise $K_{0}=1$ ) and $\alpha\left(1-\beta_{2}\right)(1-m)=m(1-\alpha)\left(1-\beta_{1}\right)$, where the variable $\beta_{2}$ is expressed through $\beta_{1}=\beta$ by the formula

$$
\beta_{2}=1-(1-\beta) \cdot \frac{1-\alpha}{\alpha} \cdot \frac{m}{1-m} .
$$

Thus, substituting (17) to the expression for $K_{0}$, we get

$$
K_{0}(\beta, \alpha)=\frac{\beta+\alpha-\alpha \beta-m}{1-m} .
$$

One can see, that (17) and inequality $\beta_{1}+\beta_{2} \leq 1$ imply that $(\beta, \alpha) \in[0,1) \times(0,1)$ should obey the inequality $(1-m) \beta \alpha \leq m(1-\beta)(1-\alpha)$. Let

$$
G_{m}=\{(\beta, \alpha) \in[0,1) \times(0,1):(1-m) \beta \alpha \leq m(1-\beta)(1-\alpha)\} .
$$

Then $G_{m} \neq \emptyset$ for any $m \in(0,1)$.

Let us now analyze the conditions (9), that can be rewritten in the form:

$$
\left\{\begin{array}{l}
m \mu_{f}(A)+(1-m) \mu_{f}(B) \leq \alpha \mu_{f}(A)+(1-\alpha) \mu_{f}(B), \\
m \mu_{f}(A)+(1-m) \mu_{f}(B) \leq \beta \mu_{f}(A)+\beta_{2} \mu_{f}(B)+\left(1-\beta-\beta_{2}\right) \mu_{f}(A \cup B),
\end{array}\right.
$$

where $\beta_{2}$ can be obtained by the formula (17). The above system can be transformed to

$$
\left\{\begin{array}{l}
(m-\alpha)\left(\mu_{f}(A)-\mu_{f}(B)\right) \leq 0 \\
(m-\beta)\left(\mu_{f}(A)-\mu_{f}(B)\right) \leq\left((1-\beta) \cdot \frac{1-\alpha}{\alpha} \cdot \frac{m}{1-m}-\beta\right)\left(\mu_{f}(A \cup B)-\mu_{f}(B)\right) .
\end{array}\right.
$$


Let us consider two cases: 1) $\left.\mu_{f}(A)=\mu_{f}(B) ; 2\right) \mu_{f}(A) \neq \mu_{f}(B)$.

If $\mu_{f}(A)=\mu_{f}(B)$, then all conditions of system (19) are fulfilled and $\bar{K}_{i n}^{D}(F) \geq \sup \left\{K_{0}(\beta, \alpha):(\beta, \alpha) \in G_{m}\right\}=1$. This value is achieved, for example, in the point $(1,0) \in \overline{G_{m}}$.

Suppose now that $\mu_{f}(A) \neq \mu_{f}(B)$. It suffices to consider one case due to the symmetry of the problem. Let, for example, $\mu_{f}(A)<\mu_{f}(B)$. Then (19) is equivalent to the system

$$
\left\{\begin{array}{l}
m \geq \alpha, \\
\beta-m \leq C_{A, B}\left((1-\beta) \cdot \frac{1-\alpha}{\alpha} \cdot \frac{m}{1-m}-\beta\right),
\end{array}\right.
$$

where $C_{A, B}=\frac{\mu_{f}(A \cup B)-\mu_{f}(B)}{\mu_{f}(B)-\mu_{f}(A)} \geq 0$ (the last inequality follows from $\mu_{f}$ monotonicity). Assume that $D_{m}$ denotes the set of points $(\beta, \alpha) \in[0,1) \times(0,1)$ that obey (20). Then $\bar{K}_{i n}^{D}(F) \geq \sup \left\{K_{0}(\beta, \alpha):(\beta, \alpha) \in G_{m} \cap D_{m}\right\}=\lim _{\varepsilon \rightarrow+0} K_{0}\left(1-\varepsilon, \varepsilon^{2}\right)=1$. The proposition is proved.

Thus, the Proposition 6 proves that the upper bound of the internal conflict of the bifocal evidence will be maximum (i.e. $\bar{K}_{i n}^{D}(F)=1$ ) if its focal elements are disjoint.

However, the situation will be completely different for bifocal evidence with intersecting focal elements. Consider the following examples.

Example 5. We have $\bar{K}_{i n}^{D}(F)=0$ for body of evidence $F=(1-m) F_{\left\{x_{1}, x_{2}\right\}}+m F_{\left\{x_{2}, x_{3}\right\}}, m \in(0,1)$, defined on $X=\left\{x_{1}, x_{2}, x_{3}\right\}$.

Example 6. We have $\bar{K}_{i n}^{D}(F)=1-m \mu_{f}\left(\left\{x_{2}, x_{3}\right\}\right)$ for body of evidence $F=(1-m) F_{\left\{x_{2}\right\}}+m F_{\left\{x_{2}, x_{3}\right\}}, m \in(0,1)$, defined on $X=\left\{x_{1}, x_{2}, x_{3}\right\}$, and $\bar{K}_{i n}^{D}(F)=D\left(F_{1}, F_{2}\right)$, where $F_{1}=F$, $F_{2}=\left(1-m \mu_{f}\left(\left\{x_{2}, x_{3}\right\}\right)\right) F_{\left\{x_{1}\right\}}+m \mu_{f}\left(\left\{x_{2}, x_{3}\right\}\right) F_{X}$.

In the Example 5 we have the case of intersection but non-inclusion of two focal elements. The focal sets are nested in the Example 6 (the so-called consonant belief structure). The internal conflict is zero in the first case. But its upper bound is nonzero in the second case. The large weight of the 'external' focal element corresponds to the smaller internal conflict.

But if bifocal evidence is a simple evidence, then the internal conflict with respect to Dempster's decomposition will be zero as shown below.

Proposition 7. If $F=(1-m) F_{A}+m F_{X}, m \in(0,1]$, then $\bar{K}_{i n}^{D}(F)=0$ for linear imprecision index $f$.

Proof. It is easy to see that the $F$ can have only decomposition of the form $F=D\left(F_{1}, F_{2}\right)$, where $F_{i}=\left(1-\alpha_{i}\right) F_{A}+\alpha_{i} m F_{X}, \alpha_{i} \in(0,1], i=1,2$. Then $K_{0}\left(F_{1}, F_{2}\right)=0$ and conditions (5)-(7), (9) have the following form

$$
\left\{\begin{array}{l}
\alpha_{1} \alpha_{2}=m, \\
\left(\alpha_{i}-m\right)\left(1-\mu_{f}(A)\right) \geq 0, \quad \alpha_{i} \in(0,1], \quad i=1,2 .
\end{array}\right.
$$

Since $1-\mu_{f}(A) \geq 0$, then the last system is equivalent to the conditions $\alpha_{1} \alpha_{2}=m$, $\alpha_{i} \geq m, i=1,2$. This system has a number of solutions (for example, $\alpha_{1}=m, \alpha_{2}=1$ ). Therefore $\bar{K}_{i n}^{D}(F)=K_{0}\left(F_{1}, F_{2}\right)=0$. The proposition is proved. 


\section{Decomposition Approach with the Disjunctive Consensus Rule}

Let us now consider the decomposition approach to evaluating the internal conflict in which the disjunctive consensus rule is used. In this case, we will consider the condition (2) instead of the restriction (6). In addition, we will take into account the pessimistic nature of the disjunctive consensus rule. This leads to the need to consider the following constraint (see Proposition 2):

$$
f(F) \geq f\left(F_{i}\right), \quad i=1,2,
$$

where $f$ is the linear imprecision index. As a result, the evaluation of the internal conflict by decomposition method with the disjunctive consensus rule is reduced to solving the optimization problem (4) with the constraints (2), (5), (21).

Let us establish some general properties of the internal conflict found by the decomposition method using the disjunctive consensus rule. We first consider the categorical evidence.

Proposition 8. We have:
1) $\underline{K}_{i n}^{D P}\left(F_{A}\right)=0$ for $A \in 2^{X} \backslash\{\emptyset\}$;
2) $\bar{K}_{i n}^{D P}\left(F_{A}\right)=1$ for $A \in 2^{X} \backslash\{\emptyset\},|A|>1$.

Proof. 1. Let $F_{1}=F_{2}=F_{A}$. Then we have $F_{A}=D P\left(F_{1}, F_{2}\right), K_{0}\left(F_{1}, F_{2}\right)=0$ and the condition (21) is true. Therefore $\underline{K}_{i n}^{D P}\left(F_{A}\right)=0$.

2. Let $|A|>1$. Then there are two sets $B, C \in 2^{X} \backslash\{\emptyset\}$ for which $B \cup C=A$ and $B \cap C=\emptyset$. Let $F_{1}=F_{B}, F_{2}=F_{C}$. We have $F_{A}=D P\left(F_{1}, F_{2}\right), K_{0}\left(F_{1}, F_{2}\right)=1$ and the condition (21) is true: $f\left(F_{A}\right)=\mu(A) \geqslant f\left(F_{1}\right)=\mu(B), f\left(F_{A}\right)=\mu(A) \geqslant f\left(F_{2}\right)=\mu(C)$ due to the monotonicity of set function $\mu$. Therefore $\bar{K}_{i n}^{D P}\left(F_{A}\right)=1$.

Proposition 8 shows that categorical evidence (but not Dirac measures) will have the greatest uncertainty of internal conflict when using decomposition with the disjunctive consensus rule.

Some properties are convenient to consider in the framework of the TBM approach [29] (see Subsection 3.2). In this case, the upper bound will always be equal to one: $\overline{\mathcal{K}}_{i n}^{D P}(F)=1$ and this value is achieved for decomposition $F=D P\left(F, F_{\emptyset}\right)$.

Proposition 9. We have $\underline{\mathcal{K}}_{i n}^{D P}\left(F_{\emptyset}\right)=1$ for strict imprecision index $f$.

Proof. Since $0=f\left(F_{\emptyset}\right) \geqslant f\left(F_{i}\right), i=1,2$ and imprecision index $f$ is strict, then $F_{1}=\sum_{i} \alpha_{i} F_{\left\{x_{i}\right\}}+\alpha_{0} F_{\emptyset}, F_{2}=\sum_{j} \beta_{j} F_{\left\{x_{j}\right\}}+\beta_{0} F_{\emptyset}$. The condition $F_{\emptyset}=D P\left(F_{1}, F_{2}\right) \Rightarrow 1=\alpha_{0} \beta_{0}$. In the other case, we have $K_{0}\left(F_{1}, F_{2}\right)=\alpha_{0} \beta_{0}+\sum_{i \neq j} \alpha_{i} \beta_{j}=1$. Therefore $\underline{\mathcal{K}}_{i n}^{D P}\left(F_{\emptyset}\right)=1$.

The following Proposition shows that the internal conflict of the Dirac measure in the considered approach will be zero, but will have the greatest uncertainty in the sense of a generalized solution in the framework of the TBM (see Subsection 3.2).

Proposition 10. If the strict imprecision index is used in the constraints of the decomposition problem, then the following properties are valid:

1) $\bar{K}_{\text {in }}^{D P}\left(F_{\{x\}}\right)=0$ for every $x \in X$;

2) $\underline{\mathcal{K}}_{i n}^{D P}\left(F_{\{x\}}\right)=0, \overline{\mathcal{K}}_{i n}^{D P}\left(F_{\{x\}}\right)=1$ for every $x \in X$ 
Proof. 1. Since $0=f\left(F_{\{x\}}\right) \geqslant f\left(F_{i}\right), i=1,2$ and imprecision index $f$ is strict, then $F_{1}=\sum_{i} \alpha_{i} F_{\left\{x_{i}\right\}}, F_{2}=\sum_{j} \beta_{j} F_{\left\{x_{j}\right\}}$. Let $x=x_{k}$. The condition $F_{\left\{x_{k}\right\}}=D P\left(F_{1}, F_{2}\right) \Rightarrow 1=\alpha_{k} \beta_{k} \Rightarrow \alpha_{k}=\beta_{k}=1$ and $K_{0}\left(F_{1}, F_{2}\right)=\sum_{i \neq j} \alpha_{i} \beta_{j}=0$. Therefore $\bar{K}_{i n}^{D P}\left(F_{\{x\}}\right)=0$.

2. But in the generalized case we have for pair $F_{1}=F_{\{x\}}, F_{2}=F_{\emptyset}: K_{0}\left(F_{1}, F_{2}\right)=1$. But for $F_{1}=F_{2}=F_{\{x\}}$ we have $K_{0}\left(F_{1}, F_{2}\right)=0$.

Finally, we establish some estimates of internal conflict for bifocal evidence with intersecting focal elements. It turns out that the result depends on the nature of the intersection of the sets.

Proposition 11. Let $A, B \in 2^{X} \backslash\{\emptyset\}, A \cap B \neq \emptyset, m \in(0,1)$. Then we have:

1) $\underline{K}_{i n}^{D P}\left(m F_{A}+(1-m) F_{B}\right)=0$;

2) if, moreover, the conditions $A \backslash B \neq \emptyset$ and $B \backslash A \neq \emptyset$ are true, then $\bar{K}_{i n}^{D P}\left(m F_{A}+(1-m) F_{B}\right)=1$.

Proof. 1. We have $K_{0}\left(F_{1}, F_{2}\right)=0$ for pair $F_{1}=m F_{A}+(1-m) F_{B}$, $F_{2}=F_{A \cap B}$ and the condition (21) is true: $f\left(m F_{A}+(1-m) F_{B}\right)=m \mu(A)+(1-m) \mu(B) \geqslant \mu(A \cap B)=f\left(F_{2}\right)$.

2. We have $K_{0}\left(F_{1}, F_{2}\right)=1$ for the following pair $F_{1}=m F_{A \backslash B}+(1-m) F_{B \backslash A}, F_{2}=F_{A \cap B}$ and the condition (21) is true: $f\left(m F_{A}+(1-m) F_{B}\right)=m \mu(A)+(1-m) \mu(B) \geqslant m \mu(A \backslash B)+(1-m) \mu(B \backslash A)=f\left(F_{1}\right)$.

The following example shows that the upper estimation of internal conflict can be arbitrarily small (see Proposition 11) in the case when one focal set is contained in another (the so-called consonant structure of evidence).

Example 7. Let $X=\left\{x_{1}, x_{2}\right\}$ and $F=(1-m) F_{\left\{x_{1}\right\}}+m F_{\left\{x_{1}, x_{2}\right\}}, m \in(0,1)$. Then (see Proposition 13 below) $\underline{K}_{i n}^{D P}(F)=0$ and $\bar{K}_{i n}^{D P}(F)=m$.

The following conclusion can be made as a result of an analysis of the general properties of an internal conflict in the case of using Dempster's rule and the disjunctive consensus rule. Suppose we have two bodies of evidence, and the focal elements of one evidence are complements (or close to complements) of the focal elements of another. Suppose we evaluate the internal conflict by the decomposition method in one case using Dempster's rule and using the disjunctive consensus rule in the other case. Then we get close conflict estimates.

\section{Decompositional Assessment of Internal Conflict for $|X|=2$}

\subsection{Decompositional Assessment with Dempster's Rule}

In this section we will evaluate internal conflict for bodies of evidence $F$ defined on $X=\left\{x_{1}, x_{2}\right\}$ using their decompositions based on Dempster's rule and canonical conflict measure (4). In this case the body of evidence $F$ has a form $F=m_{0} F_{X}+m_{1} F_{\left\{x_{1}\right\}}+m_{2} F_{\left\{x_{2}\right\}}$, where $\mathbf{m}=\left(m_{i}\right)_{i=0}^{2} \in S_{3}$. The conflict measure (and its boundaries) depends on only two parameters: $\underline{K}_{i n}^{D}(F)=\underline{K}_{i n}^{D}\left(m_{1}, m_{2}\right)$, $\bar{K}_{i n}^{D}(F)=\bar{K}_{i n}^{D}\left(m_{1}, m_{2}\right)$. Since $\underline{K}_{i n}^{D}(F)=0$, then we'll find $\bar{K}_{i n}^{D}(F)$. 
Proposition 12. Let $F=m_{0} F_{X}+m_{1} F_{\left\{x_{1}\right\}}+m_{2} F_{\left\{x_{2}\right\}} \in \mathcal{F}(X),|X|=2$. Then we have

$$
\bar{K}_{i n}^{D}(F)=\frac{m_{1} m_{2}}{\left(1-m_{1}\right)\left(1-m_{2}\right)}=\frac{m_{1} m_{2}}{\left(m_{0}+m_{1}\right)\left(m_{0}+m_{2}\right)}
$$

for $m_{0} \neq 0$ and we have $\bar{K}_{\text {in }}^{D}(F)=1$ for $m_{0}=0$.

Proof. We should find the largest value of the function (10) with constraints (11), (12), (14), (15) for computing of $\bar{K}_{i n}^{D}(F)$. We will find the decomposition into two bodies of evidence in the form $F_{1}=\alpha_{0} F_{X}+\alpha_{1} F_{\left\{x_{1}\right\}}+\alpha_{2} F_{\left\{x_{2}\right\}}, F_{2}=\beta_{0} F_{X}+\beta_{1} F_{\left\{x_{1}\right\}}+\beta_{2} F_{\left\{x_{2}\right\}}$, where $\left(\alpha_{i}\right)_{i=0}^{2} \in S_{3},\left(\beta_{i}\right)_{i=0}^{2} \in S_{3}$. We have $K_{0}\left(g_{1}, g_{2}\right)=\alpha_{1} \beta_{2}+\alpha_{2} \beta_{1}$. The conditions (11), (12), (14), (15) take the following form

$$
\begin{gathered}
\left(1-\alpha_{i}\right)\left(1-\beta_{i}\right)=\left(1-\alpha_{1} \beta_{2}-\alpha_{2} \beta_{1}\right)\left(1-m_{i}\right), \quad i=1,2, \\
\alpha_{1}+\alpha_{2} \leq m_{1}+m_{2}, \quad \beta_{1}+\beta_{2} \leq m_{1}+m_{2}, \\
\alpha_{1} \beta_{2}+\alpha_{2} \beta_{1}<1, \quad \alpha_{i} \geq 0, \quad \beta_{i} \geq 0, \quad i=1,2 .
\end{gathered}
$$

Let $\Omega=\left\{\left(\alpha_{1}, \alpha_{2}\right) \in[0,1]^{2}: \alpha_{1}+\alpha_{2} \leq m_{1}+m_{2}\right\}$. We will find the solution of the system (23) with respect to variables $\beta_{1}, \beta_{2}$. The determinant $\Delta\left(\alpha_{1}, \alpha_{2}\right)$ of this system is as follows

$$
\Delta\left(\alpha_{1}, \alpha_{2}\right)=\left(1-\alpha_{1}\right)\left(1-\alpha_{2}\right)-\left(1-m_{2}\right) \alpha_{1}\left(1-\alpha_{1}\right)-\left(1-m_{1}\right) \alpha_{2}\left(1-\alpha_{2}\right) .
$$

Note that $\Delta\left(\alpha_{1}, \alpha_{2}\right) \geq 0$ in $\Omega$. In the first instance, we consider the case $\Delta\left(\alpha_{1}, \alpha_{2}\right)>0$ in $\Omega$, or equivalently $m_{0}=1-m_{1}-m_{2}>0$.

Then we have

$$
\beta_{i}\left(\alpha_{1}, \alpha_{2}\right)=\frac{1}{\Delta\left(\alpha_{1}, \alpha_{2}\right)}\left(m_{i}-\alpha_{i}+\alpha_{i} m_{3-i}-\alpha_{3-i} m_{i}\right), \quad i=1,2 .
$$

Now the restrictions (24) define the following subset of the set $\Omega$

$$
\Omega_{0}=\left\{\left(\alpha_{1}, \alpha_{2}\right) \in[0,1]^{2} \backslash\{(1,0),(0,1)\}:\left(1-m_{3-i}\right) \alpha_{i}+m_{i} \alpha_{3-i} \leq m_{i}, i=1,2\right\} .
$$

Finally we get the following optimization problem

$$
K_{0}=\frac{\alpha_{1} \beta_{2}\left(\alpha_{1}, \alpha_{2}\right)+\alpha_{2} \beta_{1}\left(\alpha_{1}, \alpha_{2}\right)}{\Delta\left(\alpha_{1}, \alpha_{2}\right)} \rightarrow \max ,\left(\alpha_{1}, \alpha_{2}\right) \in \Omega_{0} .
$$

The point $\left(\alpha_{1}^{0}, \alpha_{2}^{0}\right)$, where

$$
\alpha_{i}^{0}=1-\frac{\sqrt{1-m_{i}}}{\sqrt{1-m_{1}}+\sqrt{1-m_{2}}-\sqrt{1-m_{1}-m_{2}}}, i=1,2,
$$

is a unique stationary point of the function $K_{0}$ in $\Omega_{0}$. This is a saddle point. The solution of the optimization problem is achieved on a border of the set $\Omega_{0}$ and

$$
\bar{K}_{\text {in }}^{D}=K_{0}\left(0, \frac{m_{2}}{1-m_{1}}\right)=K_{0}\left(\frac{m_{1}}{1-m_{2}}, 0\right)=\frac{m_{1} m_{2}}{\left(1-m_{1}\right)\left(1-m_{2}\right)} .
$$

If $m_{0}=0$, then the problem $K_{0}=\alpha_{1} \beta_{2}+\alpha_{2} \beta_{1} \rightarrow \max$ with constrains (23),(24) has a solution $\bar{K}_{i n}^{D}=1$. 
Let us analyze the result of Proposition 12. First we find the conditions when the $\bar{K}_{\text {in }}^{D} \approx 1$ is true. The formula (22) implies that the $\bar{K}_{\text {in }}^{D} \approx 1$ is true, if $m_{0} \ll \min \left\{m_{1}, m_{2}\right\}$ or $m_{0}=0$ (in the last case $\bar{K}_{\text {in }}^{D}=1$ ). For example, if the conditions $m_{0} \approx 0$ and $\min \left\{m_{1}, m_{2}\right\} \gg 0$ are met, then the corresponding belief function will be close to a probability measure. The uncertainty of estimating the internal conflict will be maximum in this case: $\underline{K}_{i n}^{D}=0, \bar{K}_{i n}^{D} \approx 1$. Under this condition the value $\bar{K}_{i n}^{D}$ is larger when the distance $\left|m_{1}-m_{2}\right|$ is less for one and the same value of $m_{0}$.

On the other hand, we have $\bar{K}_{\text {in }}^{D} \approx 0$ (and hence $K_{i n}^{D} \approx 0$ ), if the evidence is either close to the vacuous evidence $F_{X}\left(m_{0} \approx 1\right)$ or it is close to the simple (but not categorical) evidence $F_{\left\{x_{i}\right\}}^{m_{0}}=\left(1-m_{0}\right) F_{\left\{x_{i}\right\}}+m_{0} F_{X}, m_{0} \in(0,1]$.

These conclusions are quite consistent with the intuitive ideas about the internal conflict of evidence.

\subsection{Decompositional Assessment with the Disjunctive Consensus Rule}

Suppose that now the internal conflict is estimated by the decomposition method using the disjunctive consensus rule and in the case of $X=\left\{x_{1}, x_{2}\right\}$. External conflict is still calculated by the formula (1). We have the following Proposition.

Proposition 13. If $F=m_{0} F_{X}+m_{1} F_{\left\{x_{1}\right\}}+m_{2} F_{\left\{x_{2}\right\}} \in \mathcal{F}(X),|X|=2$ and $m_{1} \neq 0, m_{2} \neq 0$, then the decomposition problem (2), (4) (5), (21) has a solution iff $\sqrt{m_{1}}+\sqrt{m_{2}} \leq 1$ and in this case we have

$$
\underline{K}_{i n}^{D P}(F)=2 \sqrt{m_{1} m_{2}}, \bar{K}_{i n}^{D P}(F)=m_{0}=1-m_{1}-m_{2} .
$$

Proof. We will find the decomposition into two bodies of evidence in the form $F_{1}=\alpha_{0} F_{X}+\alpha_{1} F_{\left\{x_{1}\right\}}+\alpha_{2} F_{\left\{x_{2}\right\}}, F_{2}=\beta_{0} F_{X}+\beta_{1} F_{\left\{x_{1}\right\}}+\beta_{2} F_{\left\{x_{2}\right\}}$. The conditions (2), (11), (21) take the following form

$$
\begin{gathered}
m_{1}=\alpha_{1} \beta_{1}, \quad m_{2}=\alpha_{2} \beta_{2}, \\
\alpha_{1}+\alpha_{2} \geq m_{1}+m_{2}, \quad \beta_{1}+\beta_{2} \geq m_{1}+m_{2}, \\
\alpha=\left(\alpha_{i}\right)_{i=0}^{2} \in S_{3}, \quad \beta=\left(\beta_{i}\right)_{i=0}^{2} \in S_{3} .
\end{gathered}
$$

We will find the lower $\underline{K}_{i n}^{D P}$ and upper $\bar{K}_{i n}^{D P}$ boundaries of the internal conflict by finding the minimum and maximum of the function $K_{0}\left(F_{1}, F_{2}\right)=\alpha_{1} \beta_{2}+\alpha_{2} \beta_{1}$ with constraints (25)-(27). Further we will assume that $m_{1} \neq 0, m_{2} \neq 0$. In this case, the solution of this problem is reduced to the solution of the problem of minimizing (maximizing) the function

$$
K_{0}=\frac{\alpha_{1}}{\alpha_{2}} m_{2}+\frac{\alpha_{2}}{\alpha_{1}} m_{1}
$$

on the set

$$
\Omega_{1}\left(m_{1}, m_{2}\right)=\left\{\left(\alpha_{1}, \alpha_{2}\right) \in(0,1]^{2}: \alpha_{1}+\alpha_{2} \leq 1, \frac{m_{1}}{\alpha_{1}}+\frac{m_{2}}{\alpha_{2}} \leq 1\right\} .
$$

This set $\Omega_{1}\left(m_{1}, m_{2}\right)$ will be non-empty if and only if the condition $m_{0}=1-m_{1}-m_{2} \geq 2 \sqrt{m_{1} m_{2}} \Leftrightarrow \sqrt{m_{1}}+\sqrt{m_{2}} \leq 1$ is satisfied. In this case we have $\underline{K}_{i n}^{D P}=\left(K_{0}\right)_{\min }=2 \sqrt{m_{1} m_{2}}$ and $\bar{K}_{i n}^{D P}=\left(K_{0}\right)_{\max }=m_{0}=1-m_{1}-m_{2}$. 
Let $\quad M \quad=\quad\left\{\left(m_{1}, m_{2}\right) \in \stackrel{\circ}{S}_{2}: \Omega_{1}\left(m_{1}, m_{2}\right) \neq \emptyset\right\}=$
$\left\{\left(m_{1}, m_{2}\right) \in \stackrel{\circ}{S_{2}}: \sqrt{m_{1}}+\sqrt{m_{2}} \leq 1\right\}$.
Let us analyze the result of Proposition 13. The greatest uncertainty of internal Let us analyze the result of Proposition 13 . The greatest uncertainty of internal
conflict will be for evidence $F$ close to the vacuous evidence $F_{X}\left(m_{0} \approx 1\right)$. In this case, we have $\underline{K}_{i n}^{D P}(F) \approx 0$ and $\bar{K}_{i n}^{D P}(F) \approx 1$.

On the other hand, we have $\bar{K}_{\text {in }}^{D P}(F) \approx 0$ (and consequently, $K_{i n}^{D P}(F) \approx 0$ ) $\Leftrightarrow m_{0} \approx 0 \Leftrightarrow m_{1}+m_{2} \approx 1$. Given that $\left(m_{1}, m_{2}\right) \in M$, the last approximate equality is true if $m_{1} \approx 1 \vee m_{2} \approx 1$. In other words, the corresponding belief function should be close to the Dirac measure.

The case of the least uncertainty of internal conflict is also interesting: $\underline{K}_{i n}^{D P}(F)=\bar{K}_{i n}^{D P}(F) \Leftrightarrow \sqrt{m_{1}}+\sqrt{m_{2}}=1$. In this case, the internal conflict is equal to $K_{i n}^{D P}=2 \sqrt{m_{1}}\left(1-\sqrt{m_{1}}\right), 0<m_{1}<1$. The maximum value of such a general conflict will be equal to 0.5 and will be achieved for evidence $F=\frac{1}{2} F_{X}+\frac{1}{4} F_{\left\{x_{1}\right\}}+\frac{1}{4} F_{\left\{x_{2}\right\}}$.

In addition, comparing the estimates $\bar{K}_{i n}^{D}(F)$ (see Proposition 12) and $K_{i n}^{D P}(F)$ (see Proposition 13) leads to the following inequality: $\bar{K}_{i n}^{D}\left(m_{1}, m_{2}\right)<\underline{K}_{i n}^{D P}\left(m_{1}, m_{2}\right)$ for all $\left(m_{1}, m_{2}\right) \in M$. In other words, the assessment of internal conflict by the method of decomposition with Dempster's rule is always less than the corresponding assessment using the disjunctive consensus rule. This reflects the different nature of these rules. Dempster's rule is optimistic, and the consensus rule is pessimistic.

\section{Conclusions}

The problem of decomposition of a complex body of evidence into simple, in a certain sense, bodies of evidence within the framework of the theory of evidence is considered in this article. This problem is being investigated in relation to the assessment of internal conflict of evidence. In this case, the internal conflict is estimated as an external conflict of the decomposed set of bodies of evidence. It is shown that we must introduce some restrictions on the class of decomposable bodies of evidence in order to obtain non-trivial estimates. The article discusses only the most general restrictions associated with the nature of the decomposition rules of combination. As rules of combining, Dempster's rule and the disjunctive consensus rule are considered as two polar rules. The main problem was to study the most common properties of the estimates of the internal conflict in this decompositional approach. The problem has many solutions in this general setting. Therefore, the finding of the lower and upper bounds of internal conflict is considered in this article. In particular, we have shown that the uncertainty of estimates of internal conflict for decomposition according to Dempster's rule and the disjunctive consensus rule complement each other. For example, the uncertainty of evaluating an internal conflict of vacuous evidence using decomposition with Dempster's rule will be minimal. However, this uncertainty will be maximized when using the disjunctive consensus rule.

In general decomposition with the help of the disjunctive consensus rule gives us more conservative estimates of internal conflict than those obtained using Dempster's rule.

The decomposition approach generalizes some other methods for evaluation of internal conflict. In addition, it is more universal, it allows the use of various restrictions and it has a good interpretation. 
Other restrictions on the class of the decomposable set of bodies of evidence, in addition to general restrictions arising from the nature of the combination rules, can be used for practical application of the decomposition approach.

\section{References}

[1] Bronevich A., Klir G.J., "Measures of uncertainty for imprecise probabilities: An axiomatic approach", International Journal of Approximate Reasoning, 51 (2010), 365-390.

[2] Bronevich A., Lepskiy A., "Imprecision indices: axiomatic, properties and applications", International Journal of General Systems, 44:7-8 (2015), 812-832.

[3] Bronevich A., Lepskiy A., Penikas H., "The application of conflict measure to estimating incoherence of analyst's forecasts about the cost of shares of russian companies", Procedia Computer Science, 55 (2015), 1113-1122.

[4] Bronevich A., Rozenberg I., "The contradiction between belief functions: Its description, measurement, and correction based on generalized credal sets", International Journal of Approximate Reasoning, 112 (2019), 119-139.

[5] Burger T., "Geometric views on conflicting mass functions: from distances to angles", International Journal of Approximate Reasoning, 70 (2016), 36-50.

[6] Daniel M., "Conflicts within and between Belief Functions", Computational Intelligence for Knowledge-Based Systems Design. IPMU 2010. V.6178, Lecture Notes in Computer Science, eds. Hullermeier E., Kruse R., Hoffmann F., Springer, Berlin, Heidelberg, 2010, 696-705, https://doi.org/10.1007/978-3-64214049-5_71.

[7] Dempster A.P., "Upper and lower probabilities induced by multivalued mapping", Annals of Mathematical Statistics, 38:2 (1967), 325-339, http://doi.org/10.1214/aoms/1177698950.

[8] Destercke S., Burger T., "Toward an axiomatic definition of conflict between belief functions", IEEE Transactions on Cybernetics, 43:2 (2013), 585-596.

[9] Dubois D., Prade H., "A note on measures of specificity for fuzzy sets", International Journal of General Systems, 10 (1985), 279-283.

[10] Dubois D., Prade H., "On the combination of evidence in various mathematical frameworks", Reliability Data Collection and Analysis, eds. J. Flamm and T. Luisi, Kluwer Academic Publishers, Dordrecht, 1992, 213-241.

[11] Higashi M., Klir G.J., "Measures of uncertainty and information based on possibility distributions", International Journal of General Systems, 9 (1983), 43-58.

[12] Jousselme A.-L., Grenier D., Bosse E., "A new distance between two bodies of evidence", Information Fusion, 2 (2001), 91-101.

[13] Jousselme A.-L., Maupin P., "Distances in evidence theory: Comprehensive survey and generalizations", International Journal of Approximate Reasoning, $\mathbf{5 3}$ (2012), 118-145. 
[14] Klir G.J., Uncertainty and Information: Foundations of Generalized Information Theory, Wiley, Hoboken, NJ, 2006.

[15] Lefevre E., Colot O., Vannoorenberghe P., "Belief function combination and conflict management", Information Fusion, 3:2 (2002), 149-162.

[16] "About relation between the measure of conflict and decreasing of ignorance in theory of evidence", Proceedings of the 8th conference of the European Society for Fuzzy Logic and Technology, EUSFLAT-13, Atlantis Press, Amsterdam, 2013, $355-362$.

[17] Lepskiy A., "General schemes for the combination rules of bodies of evidence and a posterior characteristics of the quality of combination", Nechetkie Sistemy i Myagkie Vychisleniya [Fuzzy Systems and Soft Computing], 9:2 (2014), 93-114 (in Russian).

[18] Lepskiy A., "On internal conflict as an external conflict of a decomposition of evidence", BELIEF 2016. V. 9861, LNAI, eds. J. Vejnarova and V. Kratochvil, Springer, Heidelberg, 2016, 25-34.

[19] Lepskiy A., "Decomposition of evidence and internal conflict", Procedia Computer Science, 122 (2017), 186-193.

[20] Liu W., "Analysing the degree of conflict among belief functions", Artificial Intelligence, 170 (2006), 909-924.

[21] Martin A., "About Conflict in the Theory of Belief Functions", BELIEF 2012. V. 164, AISC, eds. T. Denoeux and M-H. Masson, Springer, Heidelberg, 2012, $161-168$.

[22] Osswald C., Martin A., "Understanding the large family of Dempster-Shafer theory's fusion operators - a decision-based measure", Proceedings of International Conference on Information Fusion (Florence, Italy), 2006.

[23] Pichon F., Jousselme A.-L., Ben Abdallah N., "Several shades of conflict", Fuzzy Sets and Systems, 366 (2019), 63-84.

[24] Roquel A., Le Hegarat-Mascle S., Bloch I., Vincke B., "Decomposition of conflict as a distribution on hypotheses in the framework on belief functions", International Journal of Approximate Reasoning, 55:5 (2014), 1129-1146.

[25] Samet A., Lefevre E., Yahia S.B., "Integration of extra-information for belief function theory conflict management problem through generic association rules", International Journal of Uncertainty, Fuzziness and Knowledge-Based Systems, 22:4 (2014), 531-551.

[26] Schubert J., "The Internal Conflict of a Belief Function", BELIEF 2012. V. 164, AISC, eds. T. Denoeux and M-H. Masson, Springer, Heidelberg, 2012, 169-177.

[27] Sentz K., Ferson S., Combination of evidence in Dempster-Shafer theory, Report SAND 2002-0835, Sandia National Laboratories, 2002.

[28] Shafer G., A Mathematical Theory of Evidence, Princeton University Press, Princeton, NJ, 1976, 314 pp. 
[29] Smets Ph., "The combination of evidence in the transferable belief model", IEEE Pattern Analysis and Machine Intelligence, 12:5 (1990), 447-458.

[30] Yager R.R., "Entropy and specificity in a mathematical theory of evidence", International Journal of General Systems, 9:4 (1983), 249-260.

\section{Citation}

Lepskiy A.E., "Decompositional approach for evaluation of internal conflict in the framework of the evidence theory", Nechetkie Sistemy i Myagkie Vychisleniya [Fuzzy Systems and Soft Computing], 15:1 (2020), 43-63. https://doi.org/10.26456/fssc63

\section{Information about Authors}

\section{Lepskiy Alexander Evgenyevich}

Professor at Mathematics department, National Research University Higher School of Economics.

Russia, 101000, Moscow, 20 Myasnitskaya Ulitsa, HSE.E-mail: alepskiy@hse.ru 